\title{
Reliability-Aided Multiuser Detection in Time-Frequency-Domain Spread Multicarrier DS-CDMA Systems
}

\author{
Ke Yuan, Wei Liu and Lie-Liang Yang \\ School of ECS, University of Southampton \\ SO17 1BJ, United Kingdom \\ Tel: 0044-(0)23-8059 3364, Email: ky08r,w108r,1ly@ecs.soton.ac.uk \\ http://www-mobile.ecs.soton.ac.uk
}

\begin{abstract}
In this contribution we propose and study a novel multiuser detection (MUD) scheme for multicarrier directsequence code-division multiple-access systems employing both time (T)-domain and frequency $(\mathrm{F})$-domain spreading, which are referred to as the TF/MC DS-CDMA systems. Specifically, a reliability-aided MUD scheme is proposed, which consists of a linear MUD and a so-called $L$-level maximum-likelihood (ML)MUD. The linear MUD is either a joint TF-domain linear MUD or constituted by two linear MUDs, one of which is operated in the T-domain and the other one in the F-domain. The $L$ level ML-MUD is a reduced ML-MUD, which only searches in a space of size $2^{L}$ in order to find the optimum solutions for the $L$ most unreliable data bits detected by the linear MUD. In this contribution the bit error rate (BER) performance of the TF/MC DS-CDMA using the reliability-aided MUD is investigated, when communicating over additive white Gaussian noise (AWGN) channels or over frequency-selective Rayleigh fading channels. Our simulation results show that the reliability-aided MUD is capable of significantly outperforming the corresponding linear MUD. It can be shown that, provided that the signal-to-noise ratio (SNR) is sufficiently high, the reliability-aided MUD can readily obtain several decibels of SNR gain over the linear MUD at the cost of slight or moderate increase of the detection complexity.
\end{abstract}

\section{INTRODUCTION}

In high rate broadband wireless communications multicarrier CDMA has received wide attention in recent years [16]. Multicarrier CDMA employs a range of merits, such as low intersymbol interference (ISI), low implementation complexity of carrier modulation/demodulation, high-flexibility of achieving frequency diversity, etc. [1, 4]. In multicarrier CDMA there is a family of multicarrier DS-CDMA schemes, where the transmitted signals are spread in both the T-domain and Fdomain, i.e., in the TF-domain $[1,2,5,6]$. Hence, for convenience, we refer to this family of multicarrier DS-CDMA as the TF/MC DS-CDMA. Owing to employing simultaneously both the T-domain and F-domain spreading, the TF/MC DS-CDMA systems may provide us the following advantages. First, the TF/MC DS-CDMA uses low chip-rate and short T-domain and F-domain spreading sequences instead of high chip-rate and long T-domain spreading sequences employed in the conventional single-carrier direct-sequence (DS)-CDMA [7]. Hence, relatively low-rate signal processing techniques may be used for implementation of the TF/MC DS-CDMA. Second, the maximum number of users supportable by the TF/MC DSCDMA does not have to make a trade-off with the frequencydiversity achieved by using F-domain repetition [5,6]. Third, the TF/MC DS-CDMA employs a high-flexibility for supporting variable rate services [7]. Furthermore, users supported by the TF/MC DS-CDMA can be distinguished either by their Tdomain signatures or by their F-domain signatures, or by their joint TF-domain signatures. Therefore, signal detection in the TF/MC DS-CDMA can be implemented with high-flexibility, beneficial to achieving a good trade-off between complexity and BER performance $[1,7]$.

In this contribution we propose and investigate a novel reliability-aided MUD scheme for the TF/MC DS-CDMA, and study the detection performance of the proposed MUD scheme when communicating over AWGN channels or over frequency-selective Rayleigh fading channels. In more detail, the reliability-aided MUD is operated as follows: First, linear MUD is performed, where both the joint TF-domain and separate TF-domain MUDs are considered. In the context of the joint TF-domain MUD, the minimum mean-square error (MMSE)-MUD [8, 9] is investigated. By contrast, for the separate TF-domain MUD, the T-domain and F-domain MUDs can be any combination of the MMSE-MUD and zero-forcing (ZF)MUD. After the linear MUD, initial estimates to the transmitted data bits are obtained, and simultaneously, the reliabilities associated with these detected data bits are evaluated according to the approach to be detailed in our forthcoming discourse. Then, a given, say $L$, number of most unreliable data bits are identified, while the others are treated as the reliably detected data bits. Then, the $L$-level ML-MUD is carried out, which searches in a space of size $2^{L}$ for finding the optimum solutions, in order to replace the $L$ number of most unreliable data bits detected by the linear MUD. Finally, the estimates to the transmitted data bits are formed by the data bits reliably detected by the linear MUD combining with the data bits detected by the $L$-level MLMUD.

Our study and simulation results show that, by investing a reasonable complexity, the proposed reliability-aided MUD is capable of significantly outperforming the corresponding linear MUD. For example, for the TF/MC DS-CDMA schemes 
considered in this contribution, several decibels of SNR gain may usually be obtained in both AWGN and Rayleigh fading channels, when a search space of size $2^{6}$ is invoked in the reliability-aided MUD.

\section{TIME-FrequenCy-Domain SPREAD MUltiCARrier DS-CDMA}

The system structure of the TF/MC DS-CDMA considered in this contribution is the same as that in [10]. Below a brief overview is provided.

\section{A. Tranmitted signal}

The transmitter schematic of the $k$ th uplink user supported by the TF/MC DS-CDMA is shown in Fig. 1, where, for simplicity, binary phase-shift keying (BPSK) baseband modulation is assumed. As shown in Fig. 1, the binary data stream having a bit-duration $T_{b}$ is first serial-to-parallel ( $\left.\mathrm{S} / \mathrm{P}\right)$ converted to $U$ substreams, denoting that the $k$ th user transmits $U$ bits per symbol with the symbol-duration given by $T_{s}=U T_{b}$. After the $\mathrm{S} / \mathrm{P}$ conversion, the $U$ number of data bits are first spread in the F-domain using the $U$ number of $N_{f}$-length F-domain spread sequences assigned to user $k$. The F-domain spreading generates $N_{f}$ substreams corresponding to the $N_{f}$ number of subcarriers employed by the TF/MC DS-CDMA. Then, the $N_{f}$ number of subcarrier streams are spread by a common T-domain spreading waveform $c_{k}(t)$ formed by the T-domain spreading sequence $\boldsymbol{c}_{k}=\left[c_{k 0}, c_{k 1}, \ldots, c_{k\left(N_{t}-1\right)}\right]^{T} / \sqrt{N_{t}}$ assigned to the $k$ th user, where $N_{t}$ is the T-domain spreading factor. Finally, after carrying out the multicarrier modulation as shown in Fig. 1, the $k$ th user's transmitted signal is formed, which can be expressed in the complex baseband equivalent form as

$$
s_{k}(t)=\sqrt{2 P} c_{k}(t)[\boldsymbol{\omega}(t)]^{T} \boldsymbol{C}_{f}^{(k)} \boldsymbol{b}_{k}
$$

where, in addition to those terms having been described, $\boldsymbol{\omega}(t)$ accounts for the multicarrier modulation,

$$
\begin{array}{r}
\boldsymbol{\omega}(t)=\left[\exp \left(j\left[2 \pi f_{0} t+\phi_{0}^{(k)}\right]\right), \exp \left(j\left[2 \pi f_{1} t+\phi_{1}^{(k)}\right]\right),\right. \\
\left.\ldots, \exp \left(j\left[2 \pi f_{N_{f}-1} t+\phi_{N_{f}-1}^{(k)}\right]\right)\right]^{T}
\end{array}
$$

where $f_{i}$ is the frequency of the $i$ th subcarrier, while $\phi_{i}^{(k)}$ is the associated phase. In (1) $\boldsymbol{C}_{f}^{(k)}$ is for F-domain spreading of the $U$ data symbols, which is a $\left(N_{f} \times U\right)$-dimensional matrix expressed as

$$
\begin{aligned}
\boldsymbol{C}_{f}^{(k)} & =\left[\boldsymbol{c}_{f 0}^{(k)}, \boldsymbol{c}_{f 1}^{(k)}, \ldots, \boldsymbol{c}_{f(U-1)}^{(k)}\right] \\
& =\frac{1}{\sqrt{N_{f}}}\left[\begin{array}{cccc}
c_{f 0,0}^{(k)} & c_{f 1,0}^{(k)} & \ldots & c_{f(U-1), 0}^{(k)} \\
c_{f 0,1}^{(k)} & c_{f 1,1}^{(k)} & \ldots & c_{f(U-1), 1}^{(k)} \\
\vdots & \vdots & \ddots & \vdots \\
c_{f 0, N_{f}-1}^{(k)} & c_{f 1, N_{f}-1}^{(k)} & \ldots & c_{f(U-1), N_{f}-1}^{(k)}
\end{array}\right]
\end{aligned}
$$

where $\boldsymbol{c}_{f u}^{(k)}$ is for spreading of the $u$ th bit. Finally, in (1) $\boldsymbol{b}_{k}$ contains the $U$ data bits transmitted by the $k$ th user, which can be expressed as

$$
\boldsymbol{b}_{k}=\left[b_{k}[0], b_{k}[1], b_{k}[U-1]\right]^{T} .
$$

where $b_{k}[i] \in\{+1,-1\}$ and takes either value with an equal probability of 0.5 .

\section{B. Received Signal}

Let us assume that the TF/MC DS-CDMA supports $K$ synchronous users. Then, when the $K$ user signals as shown in (1) are transmitted over $K$ Gaussian or independent Rayleigh fading channels, it can be shown that the discrete observations obtained from $N_{f}$ number of chip waveform matched-filters, each of which corresponds to one of the $N_{f}$ subcarriers, can be expressed as [10]

$$
\boldsymbol{y}=\sum_{k=1}^{K} \boldsymbol{C}_{k} \boldsymbol{H}_{k} \boldsymbol{C}_{f}^{(k)} \boldsymbol{b}_{k}+\boldsymbol{n}
$$

where $\boldsymbol{y}$ is a $N_{f} N_{t}$-length observation vector and $\boldsymbol{C}_{k}$ is a $\left(N_{f} N_{t} \times N_{f}\right)$ matrix given by $\boldsymbol{C}_{k}=\boldsymbol{I}_{N_{f}} \otimes \boldsymbol{c}_{k}$, where $\otimes$ represents the Kronecker product operation. In (5) $\boldsymbol{H}_{k}=$ $\boldsymbol{I}_{N_{f}}$, when AWGN channel is considered, while $\boldsymbol{H}_{k}=$ $\operatorname{diag}\left\{h_{0}^{(k)}, h_{1}^{(k)}, \ldots, h_{N_{f}-1}^{(k)}\right\}$, when frequency-selective fading channel is considered. Note that, when frequency-selective fading channel is considered, we assume that each of the $N_{f}$ subcarriers experiences independent Rayleigh fading [4]. Finally, in (5) $\boldsymbol{n}$ is a $N_{f} N_{t}$-length Gaussian noise vector distributed with zero mean and a covariance matrix $\sigma^{2} \boldsymbol{I}_{N_{f} N_{t}}$, where $\sigma^{2}=1 / \mathrm{SNR}$ and SNR $=E_{b} / N_{0}$ denotes the SNR per bit.

It can be readily shown that (5) can be expressed in a compact form as

$$
\boldsymbol{y}=\boldsymbol{C H C} \boldsymbol{C}_{f} \boldsymbol{b}+\boldsymbol{n}
$$

where, by definition,

$$
\begin{aligned}
\boldsymbol{C} & =\left[\boldsymbol{C}_{1}, \boldsymbol{C}_{2}, \cdots, \boldsymbol{C}_{K}\right] \\
\boldsymbol{H} & =\operatorname{diag}\left\{\boldsymbol{H}_{1}, \boldsymbol{H}_{2}, \cdots, \boldsymbol{H}_{K}\right\} \\
\boldsymbol{C}_{f} & =\operatorname{diag}\left\{\boldsymbol{C}_{f}^{(1)}, \boldsymbol{C}_{f}^{(2)}, \cdots, \boldsymbol{C}_{f}^{(K)}\right\} \\
\boldsymbol{b} & =\left[\boldsymbol{b}_{1}^{T}, \boldsymbol{b}_{2}^{T}, \cdots, \boldsymbol{b}_{K}^{T}\right]^{T}
\end{aligned}
$$

The dimensions of $\boldsymbol{C}, \boldsymbol{H}, \boldsymbol{C}_{f}$ and $\boldsymbol{b}$ are $\left(N_{f} N_{t} \times N_{f} K\right)$, $\left(N_{f} K \times N_{f} K\right),\left(N_{f} K \times U K\right)$ and $(U K \times 1)$, respectively.

Additionally, according to (5), it can be readily shown that the observation vector in the context of the $n$th subcarrier can be expressed as

$$
\boldsymbol{y}_{\lambda}=\sum_{k=1}^{K} h_{\lambda}^{(k)} \boldsymbol{c}_{k} \underbrace{\frac{1}{\sqrt{N_{f}}}\left(\sum_{u=0}^{U-1} c_{f u, \lambda}^{(k)} b_{k}[u]\right)}_{\begin{array}{c}
b_{\lambda}^{(k)} \\
\lambda=0,1, \ldots, N_{f}-1
\end{array}}+\boldsymbol{n}_{\lambda},
$$




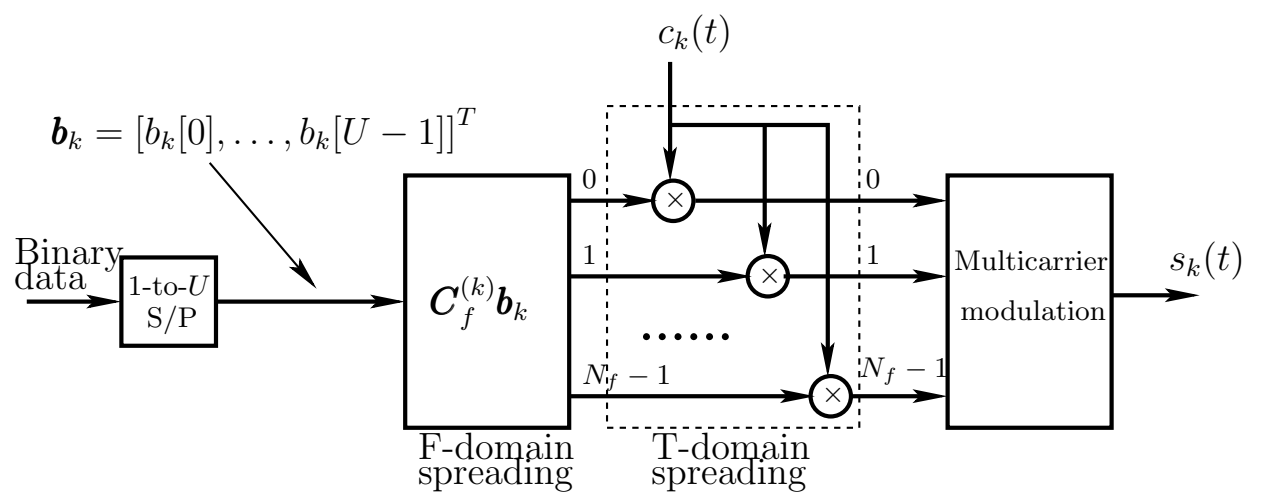

Fig. 1. Transmitter schematic block diagram of MC DS-CDMA using both time- and frequency-domain spreading.

Let define

$$
\begin{aligned}
\boldsymbol{b}_{\lambda} & =\left[b_{\lambda}^{(1)}, b_{\lambda}^{(2)}, \cdots, b_{\lambda}^{(K)}\right]^{T} \\
\boldsymbol{S} & =\left[\boldsymbol{c}_{1}, \boldsymbol{c}_{2}, \cdots, \boldsymbol{c}_{K}\right] \\
\boldsymbol{H}_{\lambda} & =\operatorname{diag}\left\{h_{\lambda}^{(1)}, h_{\lambda}^{(2)}, \cdots, h_{\lambda}^{(K)}\right\}
\end{aligned}
$$

Then, (8) can also be represented as

$$
\boldsymbol{y}_{\lambda}=\boldsymbol{S H}_{\lambda} \boldsymbol{b}_{\lambda}+\boldsymbol{n}_{\lambda}, \lambda=0,1, \ldots, N_{f}-1
$$

Note that, the representations of (8) and (10) are the convenient forms for carrying out separate TF-domain MUD [5, 6].

Let us now describe the detection algorithms proposed for the TF/MC DS-CDMA.

\section{Detection Algorithms}

The detection starts with the linear MUD carried out either jointly or separately in the TF-domain [6]. For the joint TFdomain MUD, we consider only the joint TF-domain MMSEMUD. Then, based on (6), the decision variable vector $z=$ $\left[z_{1}[0], \cdots, z_{1}[U-1] ; \cdots, z_{K}[0], \cdots, z_{K}[U-1]\right]^{T}$ for $\boldsymbol{b}$ can be expressed as $[6,9]$

$$
\boldsymbol{z}=\Re\left\{\left(\boldsymbol{C}_{f}^{T} \boldsymbol{H}^{H} \boldsymbol{C}^{T} \boldsymbol{C H} \boldsymbol{C}_{f}+\sigma^{2} \boldsymbol{I}_{K U}\right)^{-1} \boldsymbol{C}_{f}^{T} \boldsymbol{H}^{H} \boldsymbol{C}^{T} \boldsymbol{y}\right\}
$$

where $\boldsymbol{y}$ is given by (5) or (6), $\Re\{x\}$ denotes the real part of $x$.

In the context of the separate TF-domain MUD, the detection is carried out first in the T-domain and then in the F-domain. Specifically, after the T-domain MUD, the resultant variables are given by

$$
\begin{gathered}
\overline{\boldsymbol{y}}_{\lambda}=\left(\boldsymbol{H}_{\lambda}^{H} \boldsymbol{S}^{T} \boldsymbol{S} \boldsymbol{H}_{\lambda}+\rho \frac{N_{f} \sigma^{2}}{U} \boldsymbol{I}_{K}\right)^{-1} \boldsymbol{H}_{\lambda}^{H} \boldsymbol{S}^{T} \boldsymbol{y}_{\lambda}, \\
\lambda=0,1, \ldots, N_{f}-1
\end{gathered}
$$

where $\rho=0$ corresponds to using the T-domain ZF-MUD, while $\rho=1$ corresponds to using the T-domain MMSE-MUD.

Upon substituting (10) into (12), we can express $\overline{\boldsymbol{y}}_{\lambda}$ as

$$
\overline{\boldsymbol{y}}_{\lambda}=\boldsymbol{Q}_{\lambda} \boldsymbol{b}_{\lambda}+\overline{\boldsymbol{n}}_{\lambda}, \lambda=0,1, \ldots, N_{f}-1
$$

where, for convenience, we have defined

$$
\begin{gathered}
\boldsymbol{Q}_{\lambda}=\left(\boldsymbol{H}_{\lambda}^{H} \boldsymbol{S}^{T} \boldsymbol{S} \boldsymbol{H}_{\lambda}+\rho \frac{N_{f} \sigma^{2}}{U} \boldsymbol{I}_{K}\right)^{-1} \boldsymbol{H}_{\lambda}^{H} \boldsymbol{S}^{T} \boldsymbol{S} \boldsymbol{H}_{\lambda} \\
\overline{\boldsymbol{n}}_{\lambda}=\left(\boldsymbol{H}_{\lambda}^{H} \boldsymbol{S}^{T} \boldsymbol{S} \boldsymbol{H}_{\lambda}+\rho \frac{N_{f} \sigma^{2}}{U} \boldsymbol{I}_{K}\right)^{-1} \boldsymbol{H}_{\lambda}^{H} \boldsymbol{S}^{T} \boldsymbol{n}_{\lambda}
\end{gathered}
$$

Specifically, in (13), the $k$ th element of $\overline{\boldsymbol{y}}_{\lambda}$ can be expressed as

$$
\begin{array}{r}
\bar{y}_{\lambda}^{(k)}=q_{\lambda}^{(k k)} b_{\lambda}^{(k)}+I_{\lambda}^{(k)}+\bar{n}_{\lambda}^{(k)}, \lambda=0,1, \ldots, N_{f}-1 ; \\
k=1,2, \ldots, K
\end{array}
$$

where $q_{\lambda}^{(k k)}$ denotes the $(k, k)$ th element of $\boldsymbol{Q}_{\lambda}$ and $I_{\lambda}^{(k)}$ represents the residual interference, which is zero if the T-domain ZF-MUD is applied.

Let define

$$
\begin{aligned}
\tilde{\boldsymbol{y}}_{k} & =\left[\bar{y}_{0}^{(k)}, \bar{y}_{1}^{(k)}, \cdots, \bar{y}_{N_{f}-1}^{(k)}\right]^{T} \\
\tilde{\boldsymbol{n}}_{k} & =\left[I_{0}^{(k)}+\bar{n}_{0}^{(k)}, I_{1}^{(k)}+\bar{n}_{1}^{(k)}, \cdots, I_{N_{f}-1}^{(k)}+\bar{n}_{N_{f}-1}^{(k)}\right]^{T} \\
\tilde{\boldsymbol{Q}}_{k} & =\operatorname{diag}\left\{q_{0}^{(k k)}, q_{1}^{(k k)}, q_{N_{f}-1}^{(k k)}\right\}
\end{aligned}
$$

Then, we can obtain an expression for detection of $\boldsymbol{b}_{k}$ in the F-domain, which is given by

$$
\tilde{\boldsymbol{y}}_{k}=\tilde{\boldsymbol{Q}}_{k} \boldsymbol{C}_{f}^{(k)} \boldsymbol{b}_{k}+\tilde{\boldsymbol{n}}_{k}, k=1,2, \ldots, K
$$

Based on (17), the decision variable vector for $\boldsymbol{b}_{k}$ can now be formed as

$$
\begin{gathered}
\boldsymbol{z}_{k}=\Re\left\{\left[\left(\boldsymbol{C}_{f}^{(k)}\right)^{T} \tilde{\boldsymbol{Q}}_{k}^{H} \tilde{\boldsymbol{Q}}_{k} \boldsymbol{C}_{f}^{(k)}\right]^{-1}\left(\boldsymbol{C}_{f}^{(k)}\right)^{T} \tilde{\boldsymbol{Q}}_{k}^{H} \tilde{\boldsymbol{y}}_{k}\right\}, \\
k=1,2, \ldots, K
\end{gathered}
$$

for the F-domain ZF-MUD, and

$$
\begin{gathered}
\boldsymbol{z}_{k}=\Re\left\{\left(\boldsymbol{C}_{f}^{(k)}\right)^{T} \tilde{\boldsymbol{Q}}_{k}^{H}\left[\tilde{\boldsymbol{Q}}_{k} \boldsymbol{C}_{f}^{(k)}\left(\boldsymbol{C}_{f}^{(k)}\right)^{T} \tilde{\boldsymbol{Q}}_{k}^{H}+\boldsymbol{R}_{\tilde{\boldsymbol{n}}_{k}}\right]^{-1} \tilde{\boldsymbol{y}}_{k}\right\}, \\
k=1,2, \ldots, K
\end{gathered}
$$

where $\boldsymbol{R}_{\tilde{\boldsymbol{n}}_{k}}$ denotes the auto-correlation matrix of $\tilde{\boldsymbol{n}}_{k}$ in (17).

After the linear MUD (either joint or separate), the decision variables can usually be closely approximated as Gaussian 
random variables [8]. In this case, the decision variable $z_{k}[u]$ for $k=1, \ldots, K$ can be written as

$$
z_{k}[u]=b_{k}[u]+n_{k}[u], u=0,1, \ldots, U-1
$$

where $n_{k}[u]$ is approximately Gaussian distributed with mean zero and common variance, say, of $\bar{\sigma}^{2}$, which, as shown below, is not required for further processing. Finally, the estimate to $b_{k}[u]$ is decided as $\hat{b}_{k}[u]=+1$, if $z_{k}[u]>0$, while $\hat{b}_{k}[u]=-1$, otherwise.

Based on (20), the reliability of detecting $b_{k}[u]$ can be measured by the absolute of the log-likelihood ratio of $b_{k}[u]$, which can be shown is given by [11]

$$
\left|L L R_{k}[u]\right|=\frac{4}{\bar{\sigma}^{2}}\left|z_{k}[u]\right|
$$

Explicitly, the reliability for detecting $b_{k}[u]$ is simply determined by the amplitude of $z_{k}[u]$, since the factor of $4 / \bar{\sigma}^{2}$ in (21) is common to all the $K U$ data bits in $\hat{b}$. Hence, $b_{k}[u]$ can usually be detected with a higher reliability, if the corresponding amplitude of $\left|z_{k}[u]\right|$ is higher. Therefore, after the linear MUD, the receiver may enhance the detection by further making use of the information about the reliabilities of the detected $K U$ bits in $\hat{b}$.

Let after the linear MUD the $i_{0}$-th, $i_{1}$-th, $\ldots, i_{L-1}$-th bits in $\hat{b}$ be identified as the $L$ number of most unreliable bits. Then, the estimates $\hat{\boldsymbol{b}}_{L}=\left[\hat{b}_{i_{0}}, \hat{b}_{i_{1}}, \ldots, \hat{b}_{i_{L-1}}\right]^{T}$ in $\hat{\boldsymbol{b}}$ can be ignored and be replaced correspondingly by the bits in $\tilde{\boldsymbol{b}}_{L}=$ $\left\{\tilde{b}_{i_{0}}, \tilde{b}_{i_{1}}, \ldots, \tilde{b}_{i_{L-1}}\right\}$ obtained by the $L$-level ML-MUD, which finds the optimum solution according to [9]

$$
\tilde{\boldsymbol{b}}_{L}=\min _{\tilde{\boldsymbol{b}}=\hat{\boldsymbol{b}}-\hat{\boldsymbol{b}}_{L}+\boldsymbol{b}_{L} \in\{+1,-1\}^{L}}\left\{\left\|\boldsymbol{y}-\boldsymbol{C H} \boldsymbol{C}_{f} \tilde{\boldsymbol{b}}\right\|^{2}\right\}
$$

where $\tilde{\boldsymbol{b}}=\hat{\boldsymbol{b}}-\hat{\boldsymbol{b}}_{L}+\boldsymbol{b}_{L}$ means that a vector of $\tilde{\boldsymbol{b}}$ is constructed from $\hat{b}$ by replacing the bits determined by $\hat{\boldsymbol{b}}_{L}$ using the correspondingly bits in $\boldsymbol{b}_{L} \in\{+1,-1\}^{L}$. The size of the search space for $\hat{\boldsymbol{b}}_{L}$ is $2^{L}$.

Finally, it worth of noting that the complexity of the joint MMSE-MUD is $\mathcal{O}\left(K^{3} U^{3}\right)$ and that of the separate MUDs considered is $\mathcal{O}\left(U K^{3}+K U^{3}\right)$. The complexity of the reliability-aided MUD using the joint TF-domain MMSE-MUD is $\mathcal{O}\left(K^{3} U^{3}+2^{L}\right)$, while that of the reliability-aided MUD using separate TF-domain MUD is $\mathcal{O}\left(U K^{3}+K U^{3}+2^{L}\right)$.

\section{Simulation Results}

In this section a range of BER performance results are provided for characterizing the achievable BER performance of the TF/MC DS-CDMA using the proposed reliability-aided MUD, and for illustrating the trade-off between the BER performance and complexity. In our simulations the $m$-sequences were employed for both the T-domain spreading and F-domain spreading.

Figs. 2 and 3 depict the BER versus SNR per bit performance of the TF/MC DS-CDMA systems, when communicating over AWGN channels. In these two figures, the curves marked by $L=0$ represent the BER performance achieved by the

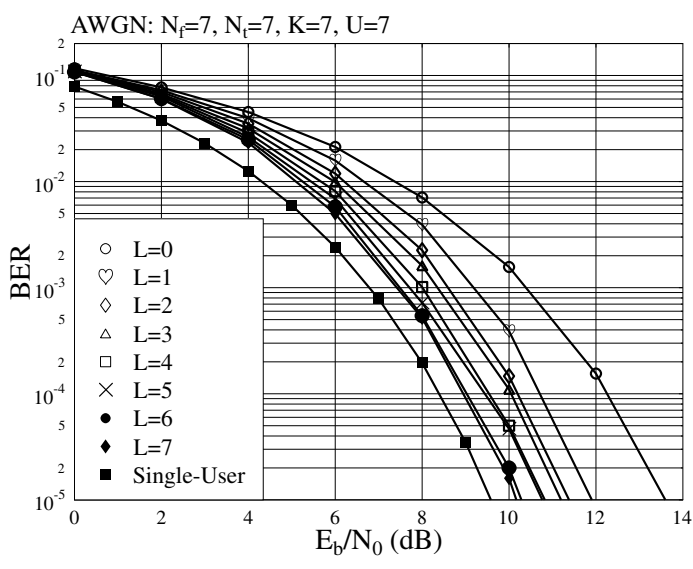

Fig. 2. BER performance of the TF/MC DS-CDMA systems employing the joint TF-domain MMSE-MUD followed by the $L$-level ML-MUD, when communicating over AWGN channels.

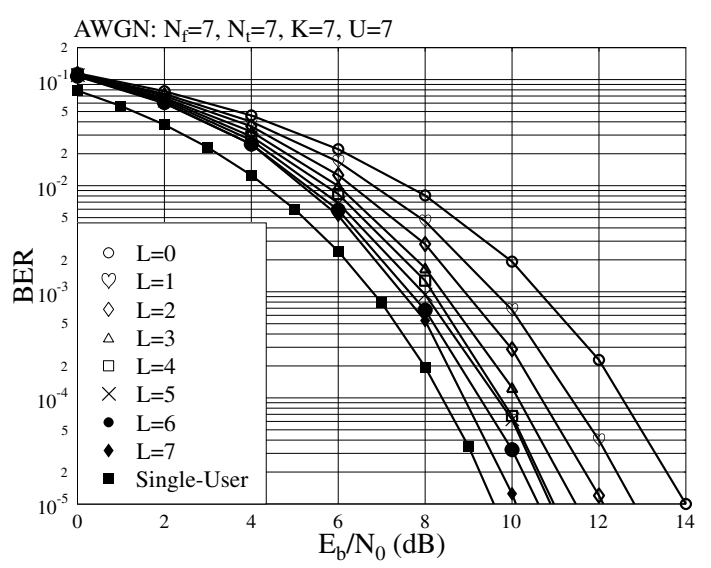

Fig. 3. BER performance of the TF/MC DS-CDMA systems employing the separate TF-domain MMSE/MMSE-MUD followed by the $L$-level ML-MUD, when communicating over AWGN channels.

corresponding linear MUDs without invoking the reliabilityaided detection. Furthermore, in the figures the single-user BER bound, which is achieved by the TF/MC DS-CDMA supporting one user, is also provided. As seen in Figs. 2 and 3, the BER performance improves, when more most unreliable data bits are invoked in the reliability-aided MUD. When $L=7$ (out of $U K=49$ ) most unreliable bits are considered, the reliability-aided MUD is capable of achieving about $4 \mathrm{~dB}$ of SNR gain over the corresponding linear MUD, for both the joint TF-domain (Fig. 2) and separate TF-domain (Fig. 3) MUDs considered.

Figs. 4 and 5 demonstrate the BER versus SNR per bit performance of the TF/MC DS-CDMA using the reliability-aided MUD, when communicating over frequency-selective Rayleigh fading channels resulting in that each subcarrier channel experiences independent fading. Again, the BER of the corresponding linear MUDs as well as the single-user BER bound are drawn 


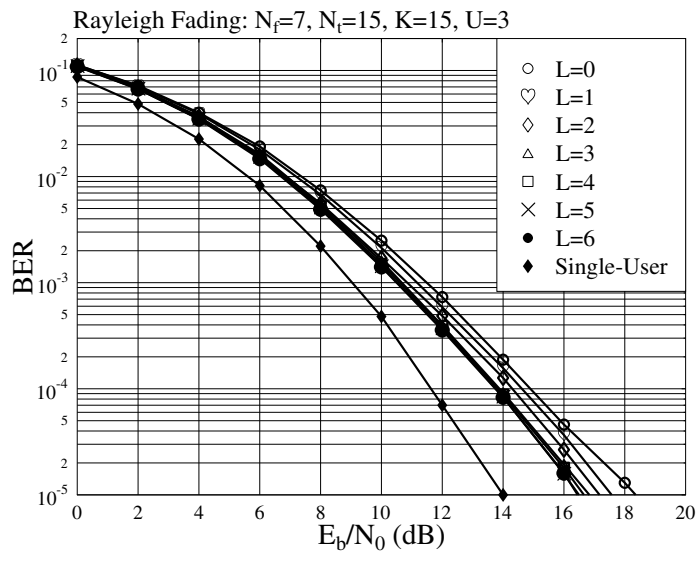

Fig. 4. BER performance of the TF/MC DS-CDMA systems employing the joint TF-domain MMSE-MUD followed by the $L$-level ML-MUD, when each subcarrier channel experiences independent Rayleigh fading.

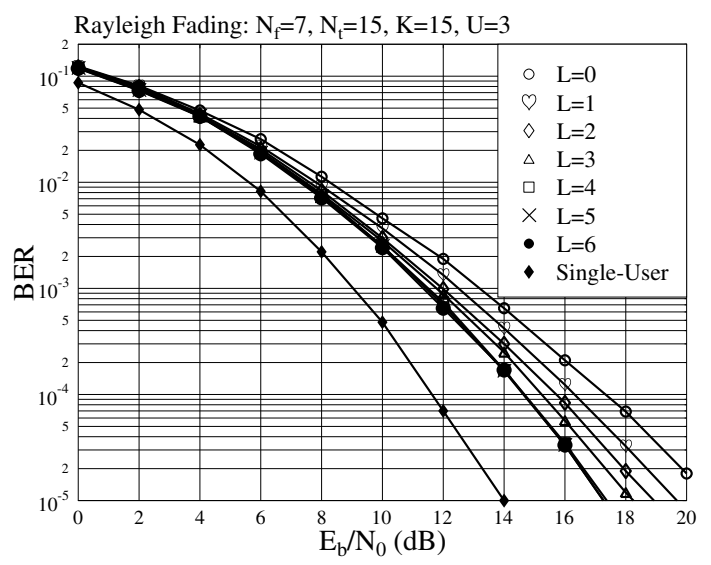

Fig. 5. BER performance of the TF/MC DS-CDMA systems employing the separate TF-domain MMSE/ZF-MUD followed by the $L$-level ML-MUD, when each subcarrier channel experiences independent Rayleigh fading.

in these figures. In Fig. 4 the joint TF-domain MMSE-MUD is employed, while in Fig. 5 the separate TF-domain MMSE/ZFMUD is employed. In comparison with the AWGN channels shown in Fig. 2, we can observe that, for the joint MMSE-MUD case, the SNR gain obtained by the reliability-aided MUD over Rayleigh fading channels is not as significant as that obtained in the AWGN channels. However, in comparison with the joint TF-domain MMSE-MUD, the SNR gain at a BER of $10^{-5}$ is still as high as $2 \mathrm{~dB}$, when the reliability-aided MUD invokes $L=6$ number of most unreliable bits for the $L$-level MLMUD. By contrast, for the separate TF-domain MMSE/ZFMUD as shown in Fig. 5, the reliability-aided MUD is also capable of achieving about $4 \mathrm{~dB}$ of SNR gain over the separate TF-domain MMSE/ZF-MUD without using reliability-aided detection.

\section{Conclusions}

In this paper we have proposed a reliability-aided MUD scheme for the TF/MC DS-CDMA and investigated the BER performance of the TF/MC DS-CDMA using this MUD scheme, when communicating over either AWGN channels or frequency-selective Rayleigh fading channels. The proposed reliability-aided MUD is constituted by a linear MUD followed by a $L$-level ML-MUD. From our study and simulation results we may conclude that the reliability-aided MUD is capable of providing a good trade-off between the affordable complexity and the achievable BER performance. Specifically, for the scenarios considered in this paper, the reliability-aided MUD may significantly outperforms its corresponding linear MUD in terms of the attainable BER performance, at the cost of slight or moderate complexity increase. Typically, the reliability-aided MUD employing a $(L=6)$-level ML-MUD is capable of achieving 2-4 dB of SNR gain over the corresponding linear MUD.

Finally, it is worth noting that the reliability-aided MUD scheme proposed in this contribution can be readily extended to many different multiple-input multiple-output (MIMO) systems, where co-channel, ISI or multiuser interference exists.

\section{ACKNOWLEDGEMENT}

The author would like to acknowledge with thanks the financial assistance from EPSRC of UK.

\section{REFERENCES}

[1] L.-L. Yang, Multicarrier Communications. Chichester, United Kingdom: John Wiley, 2009.

[2] C. You and D. Hong, "Multicarrier CDMA systems using time-domain and frequency-domain spreading codes," IEEE Transactions on Coтmunications, vol. 51, no. 1, pp. 17 - 21, January 2003.

[3] L.-C. Wang and C.-W. Chang;, "On the performance of multicarrier DSCDMA with imperfect power control and variable spreading factors," IEEE Journal on Selected Areas in Communications, vol. 24, no. 6, pp. $1154-1166$, June 2006.

[4] S. Kondo and L. B. Milstein, "Performance of multicarrier DS CDMA systems," IEEE Transactions on Communications, vol. 44, no. 2, pp. 238246, February 1996.

[5] L.-L. Yang, H. Wei, and L. Hanzo, "Multiuser detection assisted time- and frequency-domain spread multicarrier code-division multiple-access," IEEE Transactions on Vehicular Technology, vol. 55, no. 1, pp. 397-405, January 2006.

[6] L.-L. Yang and L.-C. Wang, "Zero-forcing and minimum meansquare error multiuser detection in generalized multicarrier DS-CDMA systems for cognitive radio," EURASIP Journal on Wireless Communications and Networking, vol. 2008, no. 5, Article ID 541410, doi:10.1155/2008/541410, 13 Pages, pp. -, 2008.

[7] L. L. Yang and L. Hanzo, "Multicarrier DS-CDMA: A multiple-access scheme for ubiquitous broadband wireless communications," IEEE Communications Magazine, vol. 41, no. 10, pp. 116-124, October 2003.

[8] U. Madhow and M. L. Honig, "MMSE interference suppression for direct-sequence spread-spectrum CDMA," IEEE Transactions on Communications, vol. 42, no. 12, pp. 3178-3188, December 1994.

[9] S. Verdu, Multiuser Detection. Cambridge University Press, 1998.

[10] P. Pan, L.-L. Yang, and Y. Zhang, "Spectral-efficiency of time-frequencydomain spread multicarrier DS-CDMA in frequency-selective nakagami$m$ fading channels," in IEEE VTC08 Fall. IEEE, September 2008.

[11] J. Hagenauer, E. Offer, and L. Papke, "Iterative decoding of binary block and convolutional codes," IEEE Transactions on Information Theory, vol. 42, no. 2, pp. 429-445, March 1996. 